\title{
Flow Field Simulation Research of Twin Screw Expander
}

\author{
Zhang Fan ${ }^{\mathrm{a},{ }^{*}}$, Wu Gaojie ${ }^{\mathrm{a}}$, Zhu Jun ${ }^{\mathrm{a}}$, Sun Yajing ${ }^{\mathrm{a}}$ \\ ${ }^{a}$ Mechanical Engineering College, Yangzhou University, Yangzhou, China \\ *Corresponding Author: fzhang@yzu. edu. cn
}

\begin{abstract}
When the twin screw expander working, the working medium should have a three-dimensional helical movement in internal flow field. A finite element model of expander working channel is established, based on the computational fluid dynamics theory. Then after a simulation analysis of expander internal flow field with CFX, a professional CFD simulation software, the distribution of pressure field and velocity field of the moving fluid and pressure of expander structure are gotten, which reveal the main characteristics of fluid motion in the twin screw expander. Final the dynamical mechanism of twin screw expander is defined.
\end{abstract}

Keywords: Twin Screw Expander, 3D Internal Flow Field, CFD Simulation, Pressure Field, Velocity Field.

\section{Introduction}

Twin screw expander is a fluid driven device, its core components are a couple of intermeshing screw rotor, with high-speed rotation of the screw, complete inhalation, transport and discharge of the three continuous cycle of process fluid, fluid driven transport task ${ }^{[1-3]}$. In the design phase of the rotors, in addition to considering the factors of screw strength, dimensional accuracy, tolerance, also need to assess the dynamic characteristics in the actual operation process, especially the occurrence of periodic fluid may screw rotor periodic changes in the strong resonance, which will directly affect the normal operation of double the screw pump, this paper through the ANSYS software Workbench for finite element analysis of screw constraint modal analysis, the natural frequency and vibration mode. In view of the screw rotor supported by tapered roller bearings, the constrained modal analysis to coincide with the actual constraint state, this paper adopts different constraint models to simulate the actual bearing constraints, through the analysis and comparison of the natural frequency and vibration mode, obtain more accurate analysis results to avoid the resonance of double screw pump at work. In order to achieve the purpose of optimization design of screw rotor performance.

Double screw expansion machine working process is affected by the mechanical structure and fluid motion and other factors, the internal flow field is very complex, the analytical and experimental study of mathematical analysis will consume a large amount of resources, at the same time for the expansion of machine working process is not easy to visually describe. With the development of numerical simulation technology, build a reasonable simulation model and application of computational fluid dynamics theory can not only simulate the expander in each stage of internal flow field analysis of the motion of micro changes, but also can improve the system design process, shorten the development cycle. This chapter uses the professional CFD software of twin screw expander for internal flow field numerical simulation, obtain the movement of the expander of internal flow of working medium in the expansion law of motion for internal flow, for the following expansion of power to lay the foundation for performance analysis.

\section{Twin screw expander Working Process}

\subsection{Volume Change Law of Basic Element in Expansion Process}

A pair of yin and Yang rotor slots and the shell form a space closed area, that is, the volume of the basic element, and its variation is closely related to the rotor structure parameters. In order to facilitate the calculation, the angle between the head of the inlet end face of the positive rotor and the center of the two rotor center is calculated as the angle of rotation. The case in Fig. 1 is the rotation at the intersection point of the two rotor outer circle.

Obviously, the area between the teeth of the inlet face (the shaded part in Fig. 1) is also one-to-one corresponde- 
nce, so that the relation between the intake and the tooth surfaces can be studied and studied.

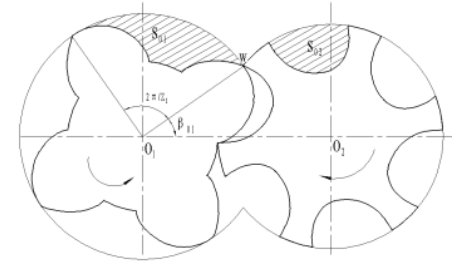

Fig. $1 \varphi_{1}=\beta_{01}$

The first stage: from. In this stage, the area between teeth is expanded from the closed volume to the maximum, and the volume of the base increases due to the gradual withdrawal of the meshing teeth.

The second stage: from. At this stage, the volume of the base remains constant as the mesh line continues to move toward the exhaust end.

The third stage: from. In this stage, the area between the teeth is gradually reduced as the teeth of the suction end meet again, and the base volume is gradually reached the maximum because of the relief of the exhaust teeth.

The fourth stage: from. In this stage, a part of the alveolus at the suction end of the positive rotor forms a new base volume with the next negative rotor tooth, and is in the suction state, and the volume of the primordium element decreases gradually due to occupation. The actual expansion at the end of the element volume for suction volume from minus.

Set the length of the micro axis of the rotor, then the relationship between the volume of the base volume on the length and the area of the end face is:

$$
d v=f\left(\varphi_{1}^{\prime}\right) d z=f\left(\varphi_{1}^{\prime}\right) d\left(\frac{T_{1}}{2 \pi} \varphi_{1}^{\prime}\right)=\frac{T_{1}}{2 \pi} f\left(\varphi_{1}^{\prime}\right) d\left(\varphi_{1}^{\prime}\right)
$$

The integral volume of the formula (1) can be obtained at the end of the rotation:

$$
V\left(\varphi_{1}^{\prime}\right)=\int_{0}^{\varphi_{1}^{\prime}} d v=\frac{T_{1}}{2 \pi} \int_{0}^{\varphi_{1}^{\prime}} f\left(\varphi_{1}^{\prime}\right) d \varphi_{1}^{\prime}
$$

From the above discussion and formula (2), the end expansion volume of the screw expander designed in this paper is:

$$
\begin{aligned}
& V_{\text {end }}=\frac{T_{1}}{2 \pi}\left(\int_{0}^{\tau_{12}+\varphi_{1 k}} f\left(\varphi_{1}^{\prime}\right) d \varphi_{1}^{\prime}-\int_{0}^{\varphi_{1 r}} f\left(\varphi_{1}^{\prime}\right) d \varphi_{1}^{\prime}\right) \\
& =\frac{T_{1}}{2 \pi}\left(2 \int_{0}^{\varphi_{1 k}} f\left(\varphi_{1}^{\prime}\right) d \varphi_{1}^{\prime}+\int_{\varphi_{1 k}}^{\tau_{1 z}} f\left(\varphi_{1}^{\prime}\right) d \varphi_{1}^{\prime}-\int_{0}^{\varphi_{r}} f\left(\varphi_{1}^{\prime}\right) d \varphi_{1}^{\prime}\right) \\
& =\frac{T_{1}}{2 \pi}\left(2 \int_{0}^{\varphi_{1 k}} \frac{f_{0}}{\varphi_{1 k}} \varphi_{1}^{\prime} d \varphi_{1}^{\prime}+\int_{\varphi_{1 k}}^{\tau_{1 z}} f_{0} d \varphi_{1}^{\prime}-\int_{0}^{\varphi_{r}} \frac{f_{0}}{\varphi_{1 k}} \varphi_{1}^{\prime} d \varphi_{1}^{\prime}\right) \\
& =\frac{T_{1}}{2 \pi} f_{0}\left(\tau_{1 z}-\frac{\varphi_{r}^{2}}{2 \phi_{1 k}}\right)=1096206874 m^{3}
\end{aligned}
$$

\subsection{Governing Equations of Fluid Motion}

The basis of computational fluid dynamics is the law of conservation of physics, which can be mathematically described by a governing equation. Fluid motion needs to satisfy three basic laws: the law of conservation of mass, the law of conservation of momentum and the law of conservation of energy. When the flow contains different components or interactions, the system follows the law of conservation of composition. When the flow is a turbulent state, the turbulent transport equation must be observed. The governing laws and transport equations can be expressed in a general form of governing equations:

$$
\frac{\partial(\rho \varphi)}{\partial t}+\operatorname{div}(\rho \mathbf{u} \varphi)=\operatorname{div}(\Gamma \cdot \operatorname{grad} \varphi)+S
$$

Formula: - density, time - velocity vector - General variables, which can represent the velocity, temperature and other solutions; - generalized diffusion coefficients generalized source terms.

Twin screw expander flow for complex turbulent flow transient three-dimensional compressible unsteady Navier-Stokes equation, while the instantaneous current can accurately describe the turbulent state by analytical method, but its solving process is very difficult, therefore, the project is widely used in Reynolds equation averaging (Reynolds-Averaged Navier-Stokes, RANS) of mean flow to solve the turbulence caused by the change of process, the equation is:

$$
\frac{\partial}{\partial t}\left(\rho u_{i}\right)+\frac{\partial}{\partial x_{j}}\left(\rho u_{i} u_{j}\right)=-\frac{\partial p}{\partial x_{i}}+\frac{\partial}{\partial x_{j}}\left[\mu \frac{\partial u_{i}}{\partial x_{j}}-\rho \overline{u_{i}^{\prime} u_{j}^{\prime}}\right]+S_{i}
$$

Type: the pressure of the fluid element, the dynamic viscosity, the velocity component, the subscript, and the superscript.

By formula (4) and formula (5), we can see that the RANS equation contains new unknown quantities, so it is necessary to introduce a new turbulence model equation to make the Reynolds equation closed.

\subsection{Flow Field Turbulence Model}

The fluid turbulence model is widely used at present is the standard model, the coefficients of the model are mainly based on the experimental results of the research objects and the researchers experience to determine, the analysis result is uncertain, the semi empirical formula is as follows:

$$
\mu_{t}=\rho C_{\mu} \frac{k^{2}}{\varepsilon}
$$

Among them: turbulent viscosity; - fluid density; empirical constant; turbulent kinetic energy; turbulent 
dissipation rate.

The assumption in the standard model for the scalar isotropic, because the rotor structure of twin screw expander is very complex, the large curvature, the internal fluid in the rotor tooth surface with non vortex flow structure of unsteady and irregular, the turbulence should be as tensor, the standard model will appear distortion.

Based on the turbulent viscosity correction, considering the influence of rotation and swirling flow in the flow, using the RNG model can better handle the flow of high strain rate and streamline the bending degree of the larger, the more close to the actual condition of internal fluid flow.

In the RNG model, the turbulent kinetic energy equations (equations) are presented:

$$
\frac{\partial(\rho k)}{\partial t}+\frac{\partial\left(\rho k u_{i}\right)}{\partial x_{i}}=\frac{\partial}{\partial x_{j}}\left[\sigma_{k} \mu_{e f f} \frac{\partial k}{\partial x_{j}}\right]+G_{k}+\rho \varepsilon
$$

Turbulent dissipation rate equation (equation):

$$
\frac{\partial(\rho \varepsilon)}{\partial t}+\frac{\partial\left(\rho \varepsilon u_{i}\right)}{\partial x_{i}}=\frac{\partial}{\partial x_{j}}\left[\sigma_{\varepsilon} \mu_{e f f} \frac{\partial \varepsilon}{\partial x_{j}}\right]+\frac{C_{1 \varepsilon}}{k} G_{k}-C_{2 \varepsilon} \rho \frac{\varepsilon^{2}}{k}
$$

Among them $\mu_{e f f}=\mu+\mu_{t} ; \mu_{t}=\rho C_{\mu} \frac{k^{2}}{\varepsilon} ; C_{\varepsilon}=0.0845$; $\alpha_{k}=\alpha_{\varepsilon}=1.39 ; C_{1 \varepsilon(2 \varepsilon)}=C_{1 \varepsilon(2 \varepsilon)}-\frac{\eta\left(1-\eta / \eta_{0}\right)}{1+\beta \eta^{3}} ; C_{1 \varepsilon}=1.42 ;$ $C_{2 \varepsilon}=1.68 ; \eta=\left(2 E_{i j} \cdot E_{i j}\right)^{1 / 2} \frac{k}{\varepsilon} ; E_{i j}=\frac{1}{2}\left(\frac{\partial u_{i}}{\partial x_{j}}+\frac{\partial u_{j}}{\partial x_{i}}\right) ; \eta_{0}=4.377 ;$ $\beta=0.012$.

Formula: dynamic viscosity coefficient - turbulent viscosity coefficient; mean velocity gradient; turbulent kinetic energy;

- empirical constants; turbulent dissipation rate and turbulent kinetic energy Prandtl number; - thermal expansion coefficient.

The RNG model not only effectively improves the computational accuracy of turbulent vortices, but also provides an analytical formula for the turbulent Prandtl number to make it more reliable in fluid flow.

\section{Modal Analysis of Internal Flow Field}

The object of CFX calculation is the fluid area between the rotor surface and the inner wall of the expander. The tooth surface of the screw rotor is very complicated,

A variety of factors of CAD system modeling and ANSYS CAD to support a variety of convenient and intuitive interface file, in order to save resources, this section based on expansion machine structure design, the modeling method of screw compressor is similar to ${ }^{[5]}$, in the Siemens NX software, three-dimensional model of the double screw expansion machine internal fluid obtained by Boolean operation, then through the format conversion into CFX.

The vector of numerical simulation and simulation analysis is finite element mesh model, and the mesh quality has the speed and quality of the solution result

Important influence. CFX pre-processing software ANSYSMeshing provides a series of powerful meshing technology, unique and advanced meshing method, which can lay a solid foundation for high-precision computing. In this paper, a comprehensive comparison of several existing ANSYS Meshing mesh method, decided to adopt the tetrahedral grid method on CFX model prior to treatment, the reasons are as follows: $1{ }^{[6]}$ tetrahedral mesh division and coordination of independent slice two algorithms can mesh for arbitrary geometry, and for the high quality requirements of the key regional grid you can use the flexible way of automatic grid refinement; 2 . rotor high speed operation, the tetrahedral partitioning method can the mesh adaptive and intelligent grid, effectively reduce the slip distortion rate, improve the convergence of grid computing. The mesh model of the completed expander flow is shown in Fig. 2. The total number of nodes is 19885 , and the total number of units is 82754

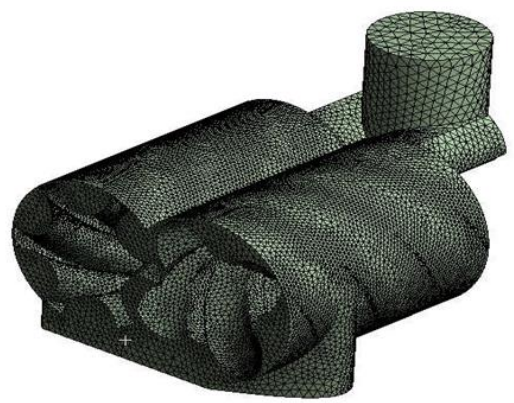

Fig. 2 mesh model of expander internal flow field

For the quality inspection standards can be divided into good mesh model through the system comes to judge the merits of ANSYS, Meshing provides the following criteria: quality inspection, inspection unit aspect ratio check, check, check Jacques ratio deviation. In this model, based on the change of curvature is larger, with deviation indicators are examined, including the abscissa deflection coefficient coefficient below 0.75 in general, said the mesh quality is good and the ordinate is the number of the coefficients of the grid. Fig. 3 is a grid skew statistical graph after checking. The grid skewness is kept below 0.75 
by the graph, which shows that the mesh model is of better quality and can be used for fluid simulation.

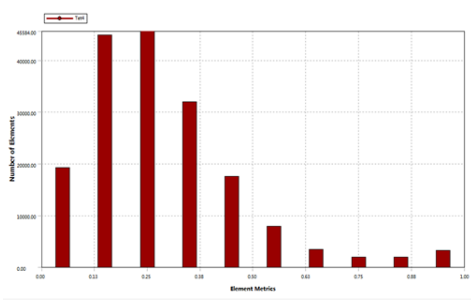

Fig. 3 skewness check of grids

\section{1 boundary condition setting}

For the development of flow field, considering the flow characteristics, the following assumptions ${ }^{[7]}: 1$. expansion as ideal fluid; 2 . working medium did not happen to the outside environment by suction, fluid leakage; 3 . exhaust orifice for isentropic flow; 4. twin screw expander working cycle is a cyclic process theory.

Considering the actual working condition of screw expander, set fluid exist in the region for the fluid domain, domain domain for the solid rotor; rotor domain motion is Rotating, in which the female rotor speed was $1000 \mathrm{r} / \mathrm{min}$, the male rotor speed $1500 \mathrm{r} / \mathrm{min}$, and set the grid deformation area, to simulate the boundary condition of movement; the default settings using the wall boundary no slip wall condition, namely, to analyze the influence of fluid flow in the inner wall of the shell; the other surface is set to a stationary wall.

The boundary condition is an important parameter to indicate the internal flow field characteristics of an expander. Because the thermodynamic characteristics of the fluid are changing at any time in the twin screw expander, the speed and temperature of the exit can not be controlled. Through the known inlet characteristics (temperature, pressure, flow rate, etc.), the outlet pressure conditions can be determined according to the internal expansion ratio of the expander, so the inlet and outlet conditions are set as pressure boundary (Pressure). The results show that the maximum recovery efficiency can be achieved when the pressure ratio matches the theoretical content ratio. Higher or lower pressure ratio will cause excessive expansion or incomplete expansion of the working medium, which will affect the performance of the expander ${ }^{[8,9]}$. The R245fa two-phase refrigerant selected in this paper sets the inlet steam temperature of $100 \mathrm{DEG} \mathrm{C}$, the pressure is $1.0 \mathrm{Mpa}$, and the outlet static pressure is $0.2 \mathrm{Mpa}$.

\section{2 solution settings}

The control parameters in the solution process need to be set in the solver, including the difference scheme setting, convergence control setting and convergence standard setting, etc.. Double screw expansion machine flow movement with time and different CFX the default format for high order model, suitable for most transient operation, the convergence control by calculating the total time; time step with automatic time scale, the system will automatically set according to the pretreatment of calculated transient simulation of single stop time scale in solution in the process of stability, so as to adjust the convergence time.

CFX in the process of solving the first category and boundary conditions according to the model initialization, the system will automatically into the initial value equation is solved by iteration, the calculation results of two times before and after subtraction, get the difference between the two, when the solution result is less than the evaluation standard, the numerical simulation is reliable. The evaluation standard has two kinds of maximum residual value and the root mean square residual value. When the general maximum residual value is $=1 \mathrm{e}-4$, the convergence effect is better, which can satisfy most engineering application calculations ${ }^{[3]}$.

\section{3 analysis of numerical simulation of internal flow field}

Double screw expansion machine working process, produced by the interaction of fluid solid coupling phenomenon between the fluid and the rotor $^{[10]}$, rotor deformation or motion in fluid loads, these changes will in turn affect the flow state of the fluid, the initial distribution and change of fluid loading, which is characterized by the coupling at the interface was two. Considering that the rotor in the expander is subjected to fluid stress, the deformation is very small and the flow field is several.

1) pressure field analysis

The rotor of the twin screw expander rotates at high speed under the action of fluid, and the fluid pressure distribution has great influence on the efficiency and performance of the expander. Research shows that the position of the stress phase angle has little effect, therefore, double screw expansion can be used at the interface of a two model to analyze the instantaneous periodic change of flow field pressure distribution machine ${ }^{[11]}$, the model below the initial phase angle of 0 degrees is analyzed.

Pressure cloud images cannot be represented by numerical values, and the pressure magnitude can only be indicated by the color depth of the feature surface. Fig. 4 is a 
double screw expansion machine flow pressure contours, can be seen from the figure, and an air inlet communicated with some red, namely inspiratory pressure, while the suction side corner change at the local pressure, analyzes its reason, this is the direction of the fluid velocity in the air flow channel is changed, the airflow pulsation, due to energy loss. With the rotation of the rotor, the rotor fluid pressure along the spiral surface gradually decreases and the pressure contour color changes slowly, until connected with the exhaust port, color will change to blue, the exhaust pressure, the pressure distribution is consistent with the actual expansion machine.

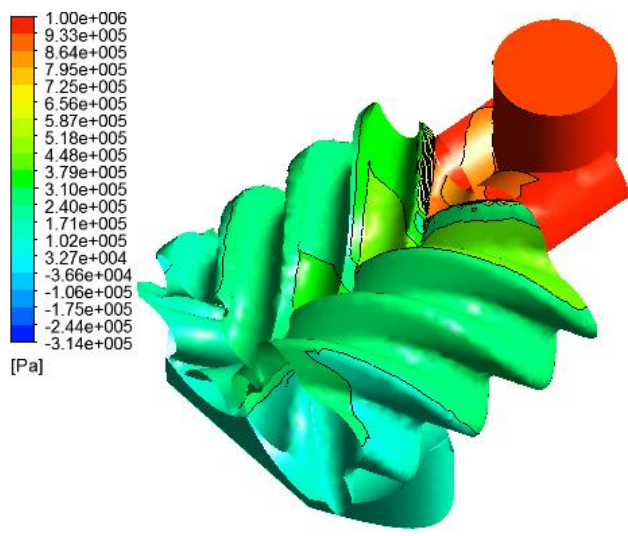

Fig. 4 Pressure nephogram of whole flow field

Double screw expansion power machine fluid in the rotor slot, push the rotor rotation, through the numerical simulation research on rotor surface pressure distribution, as shown in Fig.5, the distribution and change of flow can be obtained in the case of fluid pressure. As can be seen from the diagram, in the basic volume of the rotor, the fluid expands and decreases, the pressure decreases gradually along the spiral tooth surface of the rotor, and the pressure at different radii is also different. The element volume connected with the suction orifice of the rotor is in the suction process, and the fluid pressure gradient is larger due to the faster fluid velocity in the near suction side and the closed volume of the rotor type line selected. There are obvious rotor pressure boundary element volume, this is because a continuous space contact form the rotor tooth surface during the meshing process of sealing line caused by the expansion is a necessary condition to achieve the expansion process of the machine, or the high pressure side flow through the contact line interrupt the gap to the low voltage side leakage, greatly reduce the efficiency of expander even so, the expander failure. It is worth noting that the expansion of the machine adopts synchronous gear, screw rotors tooth surface is not in direct contact, there is the small clearance between the two meshing, the contact line of the sealing effect of the fluid is not absolute, there is still the possibility of leakage of fluid. In addition, the rotor and the inner wall of the shell has small gap, high pressure flow through the experience of the rotor tooth top leaked to an area of low pressure, thus affecting the fluid pressure field distribution, therefore, the selection of the working performance of the critical gap of the expander, the tip clearance is beneficial to the expansion engine volumetric efficiency and tooth meshing clearance reasonable improvement.

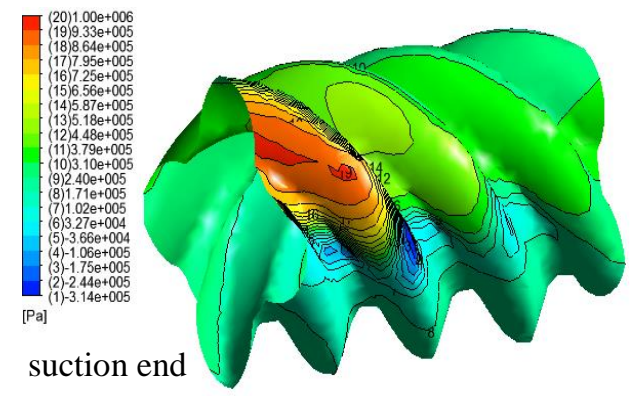

(a) Male rotor

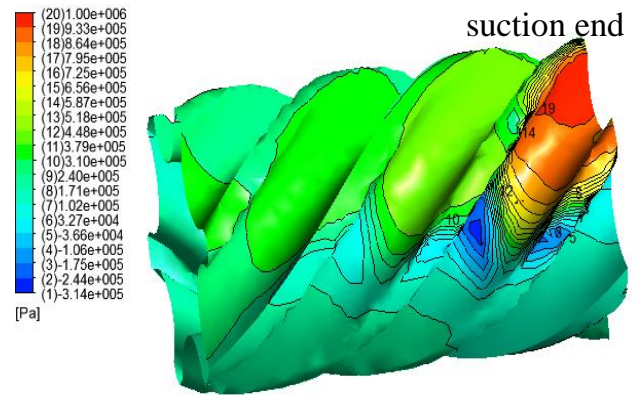

(b) Female rotor

Fig. 5 the pressure distribution on the rotor surface

In order to study the pressure distribution in the meshing area of the expander more precisely, the symmetrical section of the rotor is chosen as the pressure characteristic surface for analysis, as shown in Fig. 6. It can be seen from the figure, the element volume of fluid flow is relatively stable, the pressure is basically the same, and the adjacent element volume within the pressure along the axial direction of the rotor direction decreases gradually with the rotor; periodic suction, expansion and exhaust three working process, yin and Yang meshing region near the contact line are connected to high voltage alternating phenomenon obviously, this is decided by the expansion work principle, reflects the change of pressure expander in practical work. In addition, there is the phenomenon of negative pressure value in the meshing zone near the suction side, mainly adopts radial exhaust orifice, the exhaust angle will flow smoothly the fluid region, coupled with the regional fast leak fluid in the gap under the effect from the pressure to suction. The rotor surface pressure 
distribution to pressure variation in twin screw expander flow accurately, verify the reliability of the numerical simulation used in this paper, provides an effective method for studying the expander.
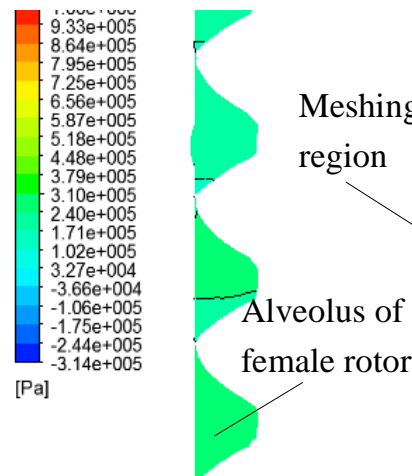

(a) axial cross section

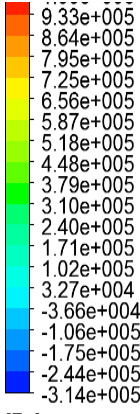

[Pa]

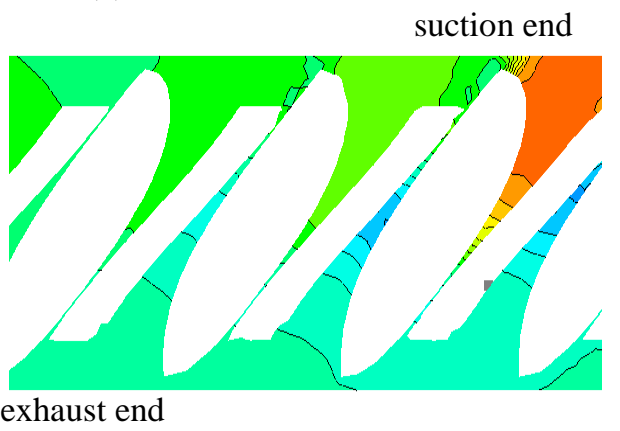

(b) radial cross section

Fig. 6 Pressure nephogram of rotor symmetrical section

Fig. 7 flow along the axial expansion average pressure curve of the length direction of the diagram, can be seen from the figure, suction cavity position change channel angle in addition to some pressure, the fluid pressure is basically the same as in other areas, namely the suction pressure; in the inspiratory phase, due to the existence of the rotor suction closed volume, the space to expand early has gradually formed a "vacuum" in the element volume, in communication with the suction port of the fluid in the moment, under the action of external pressure to quickly enter the rotor slot, so the motion space of the sudden increase of pressure fluid also occurred sudden change; in the expansion stage, the element volume enclosed area increasing in space, the internal pressure steadily decreases slowly, to exhaust pressure in the exhaust orifice. In addition, in order to make the fluid expansion in the end immediately begin to exhaust, and the exhaust passage section, maximum flow loss minimum, used in the shell along the axial radial arrangement of the exhaust orifice as hollowed out, so in the area below the central axis of the rotor in the exhaust state, its internal pressure to the pressure is smaller than the status area in the center of the rotor axis above the suction or expansion, especially in the rotor near the suction side, the gap between high and low pressure numerical contact line on both sides of the larger, which resulted in changes of the average pressure at large. Can be seen from the above analysis, has an important effect on flow structures of the suction and discharge orifice flow components of fluid flow characteristics inside machine, reasonable structural design can effectively improve the double screw expansion machine.

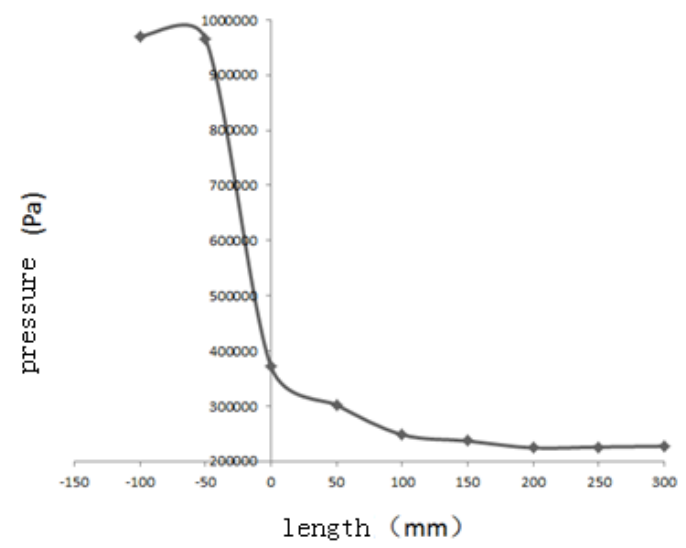

Fig. 7 axial pressure curve of the average flow

2) Velocity field analysis

Twin screw expander screw rotor rotates, the fluid flow boundary is instantaneous, at present, the problem of fluid dynamics changes continuously over time is processed by using the finite element theory, namely in the rotor of a complete period of time at a certain time of instantaneous flow field model of the selected time to represent the whole flow field model.

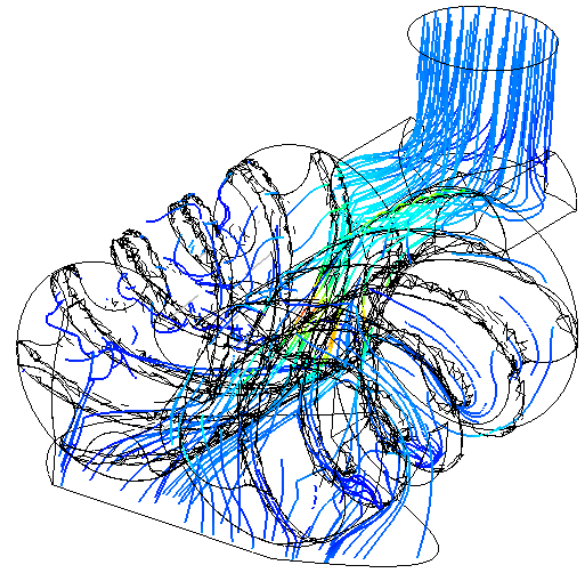

Fig. 8 Velocity streamline diagram of flow field

In order to facilitate the analysis, the flow field model of twin screw expander at 0 degrees initial phase is selected, and the flow velocity streamlines are shown in Fig. 8. Streamline is massless particles in the vector field through the whole flow path, can be seen from the figure, the fluid 
from each rotor element volume suction end to exhaust the end plane spiral movement of the rotor and the inner wall of the casing; the existence of space, close to wall very thin fluid will leak from the suction end to the exhaust end and, the fluid under the effect of rotating centrifugal force, thus the overall fluid trajectory along the circumferential direction of motion, lower curvature. The fluid moves in the opposite direction of the rotor meshing region and flows toward the exhaust end surface. The streamline can completely reflect the trajectory of fluid in the twin-screw expansion machine.

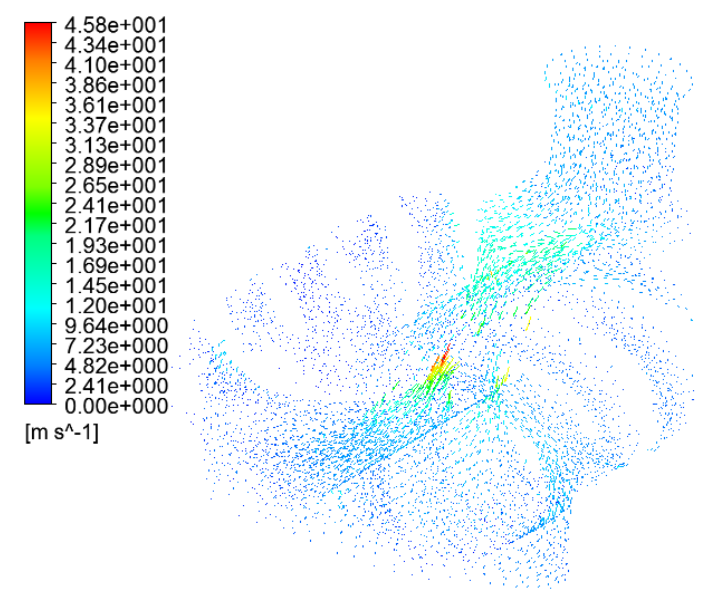

Fig. 9 Velocity vector diagram of flow field

Twin screw expander rotor to rotate under the action of the fluid, the fluid energy decreases, so the fluid experienced speed suction, expansion and exhaust after three stages decreased, Fig. 9 shows the velocity vector distribution map can show the speed variation of the flow field channels directly. On the whole, the rotor speed vector direction spiral trajectory by suction to the exhaust port of the exhaust end, fluid velocity is significantly less than the end face of the suction fluid velocity; the element volume in the Yin and Yang of the rotor surface speed is not the same, Yang top circle speed is greater than the female rotor tooth tip circle the speed of rotor teeth, this is because the structure size and speed of the male rotor than the female rotor, the difference of these parameters leads to different fluid velocity. In addition, at the junction of the rotor and rotor meshing tooth top and the inner wall of the casing, the velocity vector size is significantly different from the surrounding area, which is due to Yin and Yang rotor assembly when there is a certain gap, the element volume within a portion of the fluid through the gap will not completely expansion leakage to the low-pressure side, leading to increased flow rate.

In the working process of twin screw expander, the flow of fluid on the rotor surface is streamlined, and its velocity vector is affected by the geometric structure of the rotor and the parameters of the motion. The inspiratory phase, high pressure fluid from the suction hole into the Yin and Yang rotor slot volume, push the rotor to each other from the direction of rotation of the velocity vector changes in the rotor tooth surface; expansion stage, high pressure fluid element volume expansion between teeth under the action of pressure difference to drive the rotor to rotate at the same time. Yin and Yang of fluid in the rotor slot in the opposite direction intersect to form a vortex; the exhaust stage, the element volume decreases because of the tooth invasion, fluid velocity vector tends to be consistent, the motion of the fluid relative rules, the simulation process can clearly see the whole process of expansion machine to complete a cycle when the internal fluid velocity vector change.
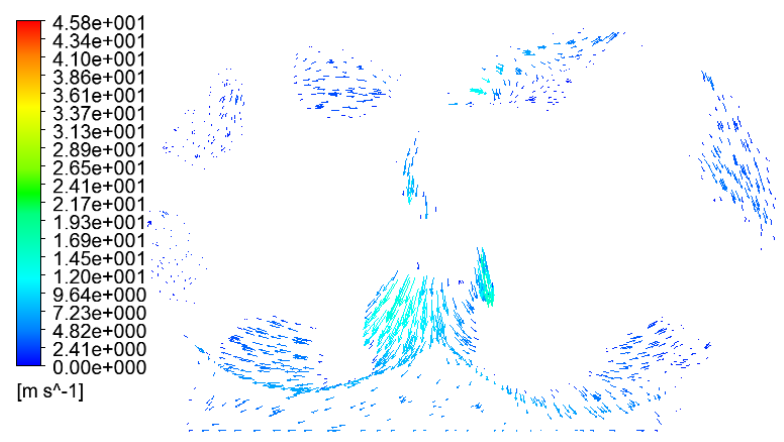

Fig. 10 velocity vector diagram of flow field section

In order to speed variation of the detailed description of twin screw expander in the working process, Fig. 10 shows the flow field velocity vector 0 degrees when the initial phase of the axial distance $150 \mathrm{~mm}$ position, the fluid is fully developed flow characteristics is more obvious. It can be seen from the figure, a very thin layer close to the inner wall of the shell is almost laminar flow, the fluid viscous force plays a dominant role, the velocity vector component along the wall normal direction varies linearly with the size distribution and flow layer thickness. The fluid expansion in the element volume, follow the rotation of the rotor to do circular motion to the rotor surface flow region, under the action of centrifugal force and the velocity vector along the tangential direction of the rotor surface; with the high-speed rotation of the rotor, the fluid in the region continues to increase, the velocity vector along the radial direction gradually from the center to the periphery of the regional differences the formation, increasing velocity vortex; regional fluid movement disorder near the rotor meshing, turbulence phenomenon, the fluid turbulence interaction force plays a dominant role, the flow condition is complicated. 
In order to explain the turbulence characteristics of the flow field of the twin-screw expander in detail, Fig. 11 shows the enlarged section of the meshing section of the above flow field. It can be seen from the figure, near the fluid turbulence near the meshing area is very complex, partly because the rotor meshing, the fluid element volume in two different velocity vector of the encounter, mixed in the rotor boundary and line structure under the restriction of the velocity vector of sudden change, the original fluid track two independent of each other, more rules are destroyed, resulting in violent turbulent motion (B); on the other hand, the contact line of the element volume is divided into high and low two areas, due to the presence of meshing clearance, fluid leakage in the pressure under the influence of the velocity vector will change, resulting in turbulence the slot (A area). The turbulent motion of twin screw expander seriously affects its performance and service life, so it is necessary to calculate the structure size and meshing clearance of the rotor reasonably.

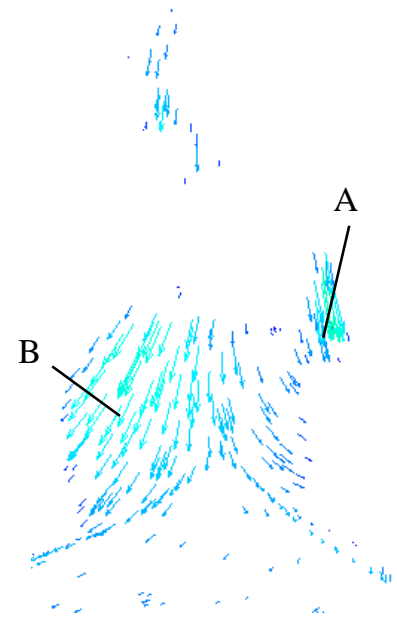

Fig. 11 enlarged drawing of flow field

\section{Conclusions}

This paper presents the mathematical model of fluid motion in twin screw expander, and shows the flow field numerical simulation method of twin screw expander in CFX software. Then after the simulation analysis, the distribution of pressure field and velocity field of the moving fluid and pressure of expander structure are obtained, which reveal the main characteristics of fluid motion in the twin screw expander. obtained the distribution of pressure and flow velocity of the rotor surface. the simulation results agree with the theoretical analysis, to a certain extent on the reliability of numerical simulation.
Thank the China Natural Science Foundation for this project. Item Number: 51375427. Thank the Jiang Su Natural Science Foundation for this project. Item Number: BY2014117-08, BY2015061-04. Thank the Jiang Su Natural Science Foundation for this project. Item Number: BY2014117-08, BY2015061-04.

\section{References}

(1) Wang. "Design theory and simulation study of medium and high pressure pure water twin screw pump", Chongqing University, 2014.

(2) Lian Hongkui, Li Yan, beam light, Li Gang, Gu Chunwei: "Review of industrial waste heat recovery and reuse technology", energy conservation technology, No. 2, pp. 123-128, 133, 2011.

(3) Xie Longhan, Zhao Xinyu: CFX fluid analysis and simulation ANSYS (Second Edition) [M]. Beijing: Electronic Industry Press, 2013.

(4) Zhai Jianhua: "General software for computational fluid dynamics (CFD)", Journal of Hebei University Of Science and Technology, No. 2, pp. 160-165, 2005.

(5) A, Kovacevic, S., Rane: "3D CFD analysis of a twin screw expander", 8th, International Conference on, Compressors and their Systems, 2013.

(6) mamtimin - Aini, Chen Hualei: "ANSYS-Workbenc 14.0 simulation technology and engineering practice", Beijing: Tsinghua University press, 2013.

(7) Wu Huiyuan, He Xueming, Dai Jin: "3D dynamic simulation and analysis research of twin screw expander based on CFD", China Mechanical Engineering, No. 24, pp. 3366-3371, 2013.

(8) I Papes, J. Degroote, J. Vierendeels: "New in twin screw expander performance for small insights scale ORC systems from 3D CFD analysis", Applied Thermal Engineering, Vol. 91, pp.535-546, 2015.

(9) Hao Tang, Huagen Wu, Xiaolin Wang, Ziwen Xing: "Performance study of a twin-screw expander used in a geothermal organic Rankine cycle power generator", Energy, Vol. 90, pp.631-642, 2015.

(10) Chen Xuefeng, Li Bing, Cao Hongrui: "Finite element method and its engineering case", Beijing: Science Press, 2014

(11) Tang Qian, Zhang Yuanxun, Gao Zhan: "Numerical simulation of flow characteristics of twin screw pump". China Mechanical Engineering, No. 12, pp.1453-1457, 2010.

\section{Acknowledgment}

